\title{
Psychometric testing of Ibadan low back pain disability questionnaire
}

\author{
Bolanle A. Nottidge ${ }^{1}$, Matthew O. Akpa ${ }^{2}$, Nse A. Odunaiya ${ }^{3}$, Adesola C. Odole ${ }^{3}$, Olufunmilayo I. \\ Fawole $^{2}$ and Aderonke O. Akinpelu ${ }^{3}$
}

Ghana Med J 2020; 54(2): 110-113 DOI: http://dx.doi.org/10.4314/gmj.v54i2.9

\author{
${ }^{1}$ Department of Physiotherapy, University of Uyo Teaching Hospital, Uyo, Akwa-Ibom State, Nigeria \\ ${ }^{2}$ Department of Epidemiology and Medical Statistics, College of Medicine, University of Ibadan, Nigeria \\ ${ }^{3}$ Department of Physiotherapy, College of Medicine, University of Ibadan, Nigeria
}

\author{
Corresponding author: Bolanle A. Nottidge $\quad$ E-mail: nottidgebolanle@yahoo.com \\ Conflict of interest: None declared
}

\begin{abstract}
SUMMARY
Background: The Ibadan Low Back Pain Disability Questionnaire (ILBPDQ) was developed to meet the need for a LBP treatment outcome measure that was appropriate for the Nigerian culture and environment. The objective of this study was to determine the psychometric properties of ILBPDQ.

Methods: This study used the validation design. One hundred and forty-two participants with LBP (LBPPs) and 142 age and sex-matched participants without LBP (NLBPPs) were included. The LBPPs were recruited consecutively from Physiotherapy clinics of selected tertiary hospitals in the six geopolitical zones of Nigeria. Both LBPPs and NLBPPs completed the ILBPDQ - the LBPPs completed the Numerical Pain Rating Scale (NPRS). The ILBPDQ was completed by 113 LBPPs two days after initial assessment. Sixty-four of the LBPPs received physiotherapy for 5-weeks, after which they were reassessed using ILBPDQ and NPRS.

Results: ILBPDQ score of LBPPs was significantly higher than that of NLBPPs (construct validity) and LBPPs NPRS score correlated significantly with their ILBPDQ score $(r=0.50)$ at baseline and post intervention $(r=0.35)$ (divergent validity). ILBPDQ scores at baseline and 48 hours later for LBPPs correlated significantly (Intra Class Correlation $=0.80$ ) (test re -test reliability). Cronbach's $\alpha$ for ILBPDQ was 0.84 (internal consistency). The postintervention ILBPDQ and NPRS scores for LBPPs were significantly lower than their pre-intervention ILBPDQ scores (responsiveness) and NPRS scores. The changes in ILBPDQ and NPRS scores of the LBPPs correlated significantly ( $\mathrm{r}=0.62)$ (responsiveness).
\end{abstract}

Conclusion: The ILBPDQ demonstrated evidence of validity, reliability and responsiveness.

Keywords: Low back pain, Functional disability, Outcome measure, Psychometric testing, Nigeria Funding: University of Ibadan Senate Research Grant.

\section{INTRODUCTION}

Low back pain (LBP) is a major cause of disability globally. ${ }^{1}$ Accurate assessment of functional disability is important for evaluating treatment and changes in LBP. Self-reported measures are important for assessment of functional disability in low back pain research. A large number of questionnaires, rating scales and outcome measures have been used to assess disability and outcome of treatment in LBP patients to facilitate clinical decision making and document treatment outcomes. ${ }^{2}$ The most commonly used are Oswestry Disability Index (ODI), Roland-Morris Disability Questionnaire (RMDQ), Million Visual Analogue Scale (MVAS), Quebec Back Pain Disability Scale (QBPDS) and Wadell Disability Index (WDI ). . $^{3-7}$
The development of the ILBPDQ, a Nigerian culture and environment-friendly scale has been described in an earlier publication. ${ }^{8}$ The objective of this study is to investigate the validity, reliability and responsiveness of ILBPDQ.

\section{METHODS}

Patients with chronic non-specific low back pain (142) recruited from the Physiotherapy out-patient clinics of tertiary hospitals in different parts of Nigeria - two tertiary hospitals in the South-West, one in the North-East, one in North-Central, one in the North-West, one in the South-East and one in the South-South, made up the LBP participants (LBPPs). 
The Non-LBP participants (NLBPPs) were 142 age and sex pairs of the LBPPs, without symptoms of LBP, recruited from patient caregivers and staff of the hospitals listed above. All participants could read and write English.

\section{Inclusion criteria}

1. The LBPPs are patients that were referred for Physiotherapy with a diagnosis of chronic non-specific low back pain, with or without radiculopathy.

2. NLBPPs were age and sex pairs of the LBPPs without symptoms of LBP, and without neuromuscular or musculoskeletal disorders of the lower limbs.

Demographic data of all participants was collected through interview. At baseline, all participants completed the ILBPDQ through interview and in addition LBPPs completed the Numerical Pain Rating Scale (NPRS). The LBPPs completed the ILBPDQ again two days after the initial scale administration. The LBPPs received Physiotherapy for five weeks, twice weekly, after which they completed the ILBPDQ and NPRS. The Physiotherapy treatment included infra-red radiation, cold or hot packs, therapeutic ultrasound, back flexion and extension exercises based on assessment using the McKenzie protocol, lumbar stabilization exercises, stretching exercises, softtissue massage, patient education, postural correction and retraining and home program.

We proposed the following hypotheses:

$\mathrm{H}_{0}=$ there would be no significant difference between the scores obtained on ILBPDQ by the LBPPs and NLBPPs. $\mathrm{H}_{1}=$ there would be a significant difference between the scores obtained on ILBPDQ by the LBPPs and NLBPPs. $\mathrm{H}_{0}=$ there would be no significant correlation between scores obtained on ILBPDQ by the LBPPs on two different occasions.

$\mathrm{H}_{1}=$ there would be a significant correlation between scores obtained on ILBPDQ by the LBPPs on two different occasions.

$\mathrm{H}_{0}=$ there would be no significant difference between the scores obtained on ILBPDQ by the LBPPs before and after a five-week physiotherapy programme.

$\mathrm{H}_{1}=$ there would be a significant difference between the scores obtained on ILBPDQ by the LBPPs before and after a five-week physiotherapy programme;

$\mathrm{H}_{0}=$ there would be no significant correlation between the changes in scores obtained on ILBPDQ and NPRS by the LBPPs after a five-week physiotherapy programme $\mathrm{H}_{1}=$ there would be significant correlation between the changes in scores obtained on ILBPDQ and NPRS by the LBPPs after a five-week physiotherapy programme.
Ethical approval was obtained for this research from the UI/UCH Ethics Committee with reference number $\mathrm{UI} / \mathrm{EC} / 11 / 0079$. Informed consent was obtained from the respondents before participating in the research.

\section{Data Analysis}

Data was summarized using mean, standard deviation and percentages for sex distribution. Mann - Whitney Utest was used to compare the scores obtained on the ILBPDQ by the LBPPs and the NLBPPs at baseline (construct validity). Spearman's correlation coefficient was used to determine the correlation between the NPRS and ILBPDQ scores of the LBPPS before treatment (divergent validity), and also between changes in ILBPDQ and NPRS scores of the LBPPs post intervention (responsiveness). Intra-class correlation coefficient (ICC) was used to determine the correlation between scores obtained on ILBPDQ by the LBPPs on two different occasions (testretest reliability). Cronbach's alpha was used to determine the item-to-item correlation of the ILBPDQ (internal consistency). Wilcoxon Signed Rank Test was used to compare the ILBPDQ and NPRS scores of the LBPPs respectively before and after 5 weeks of physiotherapy (responsiveness). The significance level was set at 0.05 .

\section{RESULTS}

A total of 142 patients with 142 matched pairs were recruited for the study. Both groups of participants (LBPPs and NLBPPs) were each made up of $69(48.6 \%)$ males and $73(51.4 \%)$ females - male:female ratio of 1:1.06.

The mean age of the LBPPs $(48.6 \pm 12.7$ years $)$ did not differ significantly from that of the NLBPPs $(48.2 \pm 12.3$ years). The median duration of LBP for the patients in the LBPPs group was 24 months (9-60 months) and $75.35 \%$ of them had pain / paraesthesia radiating to one / two lower limbs while $24.65 \%$ had pain in the low back region only. The median (Inter-quartile range - IQR) ILBPDQ score of the LBPPs [55.2 (45.6-65.6)] was significantly higher $(\mathrm{P}<0.0001)$ than that of the NLBPPs [21.4 (20-24.3)] (Table 1).

Table 1 Comparison of ILBPDQ Score of LBPPs and NLBPPs using the Mann-Whitney U Test

\begin{tabular}{|l|l|l|l|}
$\begin{array}{l}\text { LBPPs } \\
\text { Median (Range) }\end{array}$ & $\begin{array}{l}\text { NLBPPs } \\
\text { Median (Range) }\end{array}$ & z-value & p-value \\
\hline $\mathbf{5 5 . 2}$ (45.6-65.6) & $21.4(20-24.3)$ & -14.49 & 0.0001 \\
\hline
\end{tabular}

The NPRS score of the LBPPs $(6.44 \pm 1.61)$ correlated moderately $(\mathrm{r}=0.50)$ and significantly with their ILBPDQ score $(\mathrm{P}<0.0001)($ Table 2$)$. 


\section{Original Article}

Table 2 Spearman's Correlation Coefficient between ILBPDS and NPRS Score of LBPPs

\begin{tabular}{|c|c|c|c|}
\hline & $\begin{array}{l}\text { Pre-Rx NPRS } \\
\text { Score }\end{array}$ & $\begin{array}{l}\text { Post-Rx NPRS } \\
\text { Score }\end{array}$ & $\begin{array}{l}\text { Change in } \\
\text { NPRS } \\
\text { Score }\end{array}$ \\
\hline & $\mathrm{r}(\mathrm{p})$ & $\mathrm{r}(\mathrm{p})$ & $\mathrm{r}(\mathrm{p})$ \\
\hline $\begin{array}{l}\text { Pre-Rx ILBPDQ } \\
\text { Score }\end{array}$ & $0.50(\mathrm{p}=0.0000)$ & & \\
\hline $\begin{array}{l}\text { Post-Rx ILBPDQ } \\
\text { Score }\end{array}$ & & $0.35(\mathrm{p}=0.0000)$ & \\
\hline $\begin{array}{l}\text { Change in } \\
\text { ILBPDQ Score }\end{array}$ & & & $0.62(\mathrm{p}=0.000)$ \\
\hline
\end{tabular}

The median (IQR) ILBPDQ score at first visit [55.7(45.365.6)] and 48 hours later [51.8 (41.3- 61.7)] were significantly correlated $(\mathrm{ICC}=0.80, \mathrm{p}<0.05)$. The internal consistency of ILBPDQ was demonstrated by a Cronbach's alpha of 0.84 .

The median (IQR) ILBPDQ score after the 5 weeks of physiotherapy [36.5(30.0-49.4)], was significantly lower $(\mathrm{p}<0.05)$ than that before treatment [55.2 (45.6-65.6)] (Table 3). There was a significant moderate correlation $(\mathrm{r}=0.62, \mathrm{p}<0.05)$ between change in ILBPDQ score and change in NPRS score of the LBPP after 5 weeks of physiotherapy (Table 2).

Table 3 Wilcoxon Signed Rank Test for Pre \& PostTreatment of ILBPDQ and NPRS Scores of LBPPs

\begin{tabular}{|l|l|l|l|} 
Variable & $\begin{array}{l}\text { Pre-Treatment } \\
\text { Median } \\
\text { (Range) }\end{array}$ & $\begin{array}{l}\text { Post-Treatment } \\
\text { Median (Range) }\end{array}$ & z- value \\
\hline NPRS Score & $7.0(5.0-8.0)$ & $4.0(3.0-5.0)$ & \\
\hline ILBPDQ Score & $55.2(45.6-65.6)$ & $36.5(30.0-49.4)$ & -6.13 \\
\hline
\end{tabular}

\section{DISCUSSION}

The objective of this study was to investigate the psychometric properties of the ILBPDQ. The significantly higher ILBPDQ score of the LBPPs versus that of the NLBPPs suggested that ILBPDQ is able to differentiate between patients with symptoms of LBP and those without symptoms of LBP. The hypothesis that there would be no significant difference between the ILBPDQ scores of the LBPPs and NLBPPs was therefore rejected. The significantly higher ILBPDQ scores of the LBPPs also show that these participants have more functional disabilities than those individuals without symptoms of LBP - the NLBPPs, since higher scores indicate higher levels of functional disability. This provides evidence that ILBPDQ has adequate construct validity. This is similar to the findings of Hicks and Manal, who reported that participants with high pain severity and high level of functional limitation had higher scores on the modified ODI and QBPDS than other participants, which represents good construct validity for both scales. ${ }^{9}$ There was also a moderate significant correlation between ILBPDQ score and NPRS score $(r=0.50)$ at baseline.
The hypothesis that there would be no significant correlation between the ILBPDQ and NPRS scores of the LBPPs was therefore rejected. The result of this study is in agreement with other studies where there was a weak to moderate correlation between pain scores and functional disability score. ${ }^{7,10,11} \mathrm{Kopec}$ found a moderate correlation of 0.54 between a 7 - point scale of pain and QBDS. ${ }^{6}$

An intra-class correlation of 0.80 obtained in this study can be considered as good. The hypothesis that there would be no significant correlation between the ILBPDQ scores of the LBPPs on two different occasions was therefore rejected. Test-retest correlation coefficient reported for other low back questionnaires in literature vary between 0.63 and $0.99 .3,12$ High coefficients were obtained in studies where retest was carried out the same day as the first test or on the following day. Such studies include those by Hick and Manal that reported an ICC of 0.92 and 0.94 respectively for the modified ODI and QBPDS $^{13}$ and that by Roland and Morris that reported test - retest reliability correlations of 0.91 (same day) for RMDQ. ${ }^{7}$ Patients might have remembered their initial responses to the questions in these studies. Streiner and Norman recommended an interval between 2 and 14 days. ${ }^{13}$ Thus, factors like memory effect, difference in methods and population used may have contributed to the pigher ICC than that observed in this study. For evidence of internal consistency or homogeneity of items on a scale, a Cronbach"s alpha of $>0.70$ is considered as acceptable. ${ }^{14}$ The Cronbach"s alpha of 0.84 obtained in our 0.890 dy indicates an acceptable level of internal consistency of items on ILBPDQ and it provides the assurance that random errors are minimized. The acceptable level of internal consistency of ILBPDQ provides additional evidence that the scale is reliable. This result is comparable to that of Jarvikoski who reported a Cronbach alpha of 0.84 for RMDQ, and to that of Kopec, who reported 0.87 using version 2.0 of ODI. ${ }^{6,15}$ The result of this study is also similar to that of Holt et al who reported a Cronbach alpha of 0.85 for the Low Back Outcome Score and to that of Salen et al who reported a Cronbanch alpha of 0.84 for the Disability Rating Index. ${ }^{16,17}$

The finding that the ILBPDQ score of the LBPPs was significantly lower after five weeks of Physiotherapy compared to that at baseline provides evidence of adequate degree of responsiveness of ILBPDQ. The significant correlation between changes in ILBPDQ and NPRS scores of the LBPPs further supports the evidence that ILBPDQ is a responsive outcome measure. The hypothesis that there would be no significant correlation between changes in ILBPDQ and NPRS scores after 5 weeks of Physiotherapy was therefore rejected. 
This shows that ILBPDQ is able to detect changes in patients' condition or disability level over time. This implies that ILBPDQ can be used to monitor changes in patients with low back pain undergoing physiotherapy.

\section{CONCLUSION}

The Ibadan low back pain disability questionnaire is a valid, reliable and responsive outcome measure in low back pain and may be used for measuring functional disability in patients with low back pain in Nigeria and other similar clinical settings.

\section{REFERENCES}

1. Buchbinder R, Blyth FM, March LM, Brooks P, Woolf AD HD. Placing the global burden of low back pain in context. Best Pract Res Clin Rheumatol. 2013;27(5):575-589.

doi:10.1016/j.berh.2013.10.007

2. Bombardier C. Outcome assessments in the evaluation of treatment of spinal disorders: summary and general recommendations. Spine (Phila Pa 1976). 2000;25(24):3100-3103.

3. Fairbank JC, Couper J, Davies JB, O'Brien JP. The Oswestry low back pain disability questionnaire. Physiotherapy. 1980;66(8):271-273.

4. Roland M, Morris R. A study of the natural history of back pain. Part I: development of a reliable and sensitive measure of disability in low-back pain. Spine (Phila Pa 1976). 1983;8(2):141-144.

5. Million R, Hall W, Nilsen KH, Baker RD, Jayson MI. Assessment of the progress of the back-pain patient 1981 Volvo Award in Clinical Science. Spine (Phila Pa 1976). 7(3):204-212.

6. Kopec JA, Esdaile JM, Abrahamowicz M, et al. The Quebec Back Pain Disability Scale. Measurement properties. Spine (Phila Pa 1976). 1995;20(3):341352 .

7. Waddell G, Main CJ. Assessment of severity in lowback disorders. Spine (Phila Pa 1976). 1984;9(2):204-208.

8. Nottidge BA, Odole AC, Odunaiya NA, Akpa MO, Fawole OI, Akinpelu AO. Development and struc- tural validity of a Nigerian culture- and environment-friendly low back pain outcome measure: Ibadan Low Back Pain Disability Questionnaire. Ghana Med J. 2019;53(2):126-134. doi:10.4314/gmj.v53i2.7

9. Hicks GE, Manal TJ. Psychometric Properties of Commonly Used Low Back Disability Questionnaires: Are They Useful for Older Adults with Low Back Pain? Pain Med. 2009;10(1):85-94. doi:10.1111/j.1526-4637.2008.00548.x

10. McGorry RW, Shaw WS, Lin J-H. Correlations between pain and function in a longitudinal low back pain cohort. Disabil Rehabil. 2011;33(11):945-952. doi:10.3109/09638288.2010.515285

11. Duruöz MT, Özcan E, Ketenci A, Karan A. Development and validation of a functional disability index for chronic low back pain. J Back Musculoskelet Rehabil. 2013;26(1):45-54. doi:10.3233/BMR2012-00349

12. Deyo RA, Diehl AK. Measuring physical and psychosocial function in patients with low-back pain. Spine (Phila Pa 1976). 1983;8(6):635-642.

13. Streiner DL, Norman GR. Health Measurement Scales: A Practical Guide to Their Development and Use.; 2008. doi:10.1093/acprof:oso/9780199231881.001.0001

14. George, D; Mallary P. SPSS for Windows Step by Step: A Simple Guide and Reference (11.0 Update). 4th ed. Boston: Allyn and Bacon; 2003.

15. Jarvikoski A, Mellin G, Estlander A-M, et al. Outcome of Two Multimodal Back Treatment Programs With and Without Intensive Physical Training.

16. J Spinal Disord. 1993;6(2):93-98 doi:10.1097/00002517-199304000-00001

17. Holt AE, Shaw NJ, Shetty A, Greenough CG. The reliability of the Low Back Outcome Score for back pain. Spine (Phila Pa 1976). 2002;27(2):206-210.

18. Salen BA, Erik E V., Nordemar R. The disability rating index: An instrument for the assessment of disability in clinical settings. $J$ Clin Epidemiol. 1994;47(12):1423-1435. doi:10.1016/08954356(94)90086-8 